\title{
Structural evidence for enhanced polarization in a commensurate short-period $\mathrm{BaTiO}_{3} / \mathrm{SrTiO}_{3}$ superlattice
}

\author{
W. Tian, J. C. Jiang, and X. Q. Pan ${ }^{\text {a) }}$ \\ Department of Materials Science and Engineering, University of Michigan, Ann Arbor, \\ Michigan 48109-1236 \\ J. H. Haeni, Y. L. Li, L. Q. Chen, and D. G. Schlom \\ Department of Materials Science and Engineering, The Pennsylvania State University, University Park, \\ Pennsylvania 16802-5005 \\ J. B. Neaton and K. M. Rabe \\ Department of Physics and Astronomy, Rutgers University, Piscataway, New Jersey 08854-8019 \\ Q. X. Jia \\ MPA-STC, Los Alamos National Laboratory, Los Alamos, New Mexico 87545
}

(Received 10 November 2005; accepted 30 June 2006; published online 29 August 2006)

\begin{abstract}
A short-period $\left(\mathrm{BaTiO}_{3}\right)_{6} /\left(\mathrm{SrTiO}_{3}\right)_{5}$ superlattice was characterized by $\mathrm{x}$-ray diffraction and transmission electron microscopy. The superlattice is epitaxially oriented with the $c$ axes of $\mathrm{BaTiO}_{3}$ and $\mathrm{SrTiO}_{3}$ normal to the (001) surface of the $\mathrm{SrTiO}_{3}$ substrate. Despite the large in-plane lattice mismatch between $\mathrm{BaTiO}_{3}$ and $\mathrm{SrTiO}_{3}(\sim 2.2 \%)$, the superlattice interfaces were found to be nearly commensurate. The crystallographic $c / a$ ratio of the superlattice was measured and the results agree quantitatively with first-principles calculations and phase-field modeling. The agreement supports the validity of the enhanced spontaneous polarization predicted for short-period $\mathrm{BaTiO}_{3} / \mathrm{SrTiO}_{3}$ superlattices. (C) 2006 American Institute of Physics. [DOI: 10.1063/1.2335367]
\end{abstract}

Superlattices of thin ferroelectric and nonferroelectric perovskite layers have been predicted $^{1,2}$ or reported $^{3-9}$ to possess many improved physical properties over homogeneous thin films of the same compositions. Among these are reported enhancements of dielectric constants and remanent polarization in short-period $\mathrm{BaTiO}_{3} / \mathrm{SrTiO}_{3}$ superlattices. ${ }^{3,5}$ While such reports need to be carefully evaluated considering that the movement of space charge in the superlattices can spuriously produce an apparent significant enhancement of dielectric constant, ${ }^{10,11}$ the improved properties could result from the lattice strain sustained in $\mathrm{BaTiO}_{3}$ due to its $\sim 2.2 \%$ lattice mismatch with $\mathrm{SrTiO}_{3}$. ${ }^{3,5}$

Recently, Neaton and Rabe performed first-principles calculations on a series of short-period $\mathrm{BaTiO}_{3} / \mathrm{SrTiO}_{3}$ superlattices commensurately strained to an underlying $\mathrm{SrTiO}_{3}$ substrate to investigate their ground-state structures and polarizations. ${ }^{12}$ They predicted an overall enhancement of the spontaneous polarization of these ferroelectric superlattices, including the presence of a spontaneous polarization within the $\mathrm{SrTiO}_{3}$ layers. While an increase of polarization in $\mathrm{BaTiO}_{3}$ is expected, ${ }^{13}$ the prediction of unstrained $\mathrm{SrTiO}_{3}$ being tetragonal and polar is, at first, surprising. Although strain is known to induce ferroelectricity in $\mathrm{SrTiO}_{3},{ }^{14,15}$ including $\mathrm{SrTiO}_{3}$ in superlattices, ${ }^{6,7}$ thick unstrained $\mathrm{SrTiO}_{3}$ is not ferroelectric at any temperature. Neaton and Rabe, however, predicted that thin unstrained $\mathrm{SrTiO}_{3}$ layers in proximity to ferroelectric $\mathrm{BaTiO}_{3}$ polarize due to electrostatic effects. Their calculations shed light on the understanding of ferroelectric superlattices at the atomic scale.

Testing these predictions is challenging, however, due to the difficulty in fabricating such short-period superlattices with sufficient structural perfection. Although short-period $\mathrm{BaTiO}_{3} / \mathrm{SrTiO}_{3}$ superlattices have been produced by various

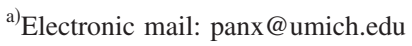

methods, convincing evidence of a high degree of structural perfection in terms of interfacial abruptness and coherency has rarely been reported. ${ }^{16,17}$

Over the last few years, significant progress has been achieved in controlling the synthesis of ferroelectric superlattices at the atomic scale using reactive molecular-beam epitaxy (MBE). ${ }^{18,19}$ In the present letter, we discuss the growth of a short-period superlattice consisting of a six-unitcell thick $\mathrm{BaTiO}_{3}$ layer followed by a five-unit-cell thick $\mathrm{SrTiO}_{3}$ layer, repeated 20 times. We abbreviate this configuration by $\left[\left(\mathrm{BaTiO}_{3}\right)_{6} /\left(\mathrm{SrTiO}_{3}\right)_{5}\right]_{20}$. X-ray diffraction (XRD) and high-resolution transmission electron microscopy (HRTEM) results are presented to demonstrate the structural perfection of this coherent superlattice with abrupt interfaces.

The $\left[\left(\mathrm{BaTiO}_{3}\right)_{6} /\left(\mathrm{SrTiO}_{3}\right)_{5}\right]_{20}$ superlattice thin film was grown by reactive $\mathrm{MBE}$ at a substrate temperature of $650{ }^{\circ} \mathrm{C}$ and an oxygen background pressure of $2 \times 10^{-7}$ Torr. $^{19,20}$ After growth the film was annealed in 1 atm of flowing oxygen at $1000{ }^{\circ} \mathrm{C}$ for $13 \mathrm{~h}$ to reduce the concentration of oxygen vacancies. HRTEM was performed in a JEOL 4000EX operated at $400 \mathrm{kV}$. The first-principles calculations are performed with the Vienna $a b$ initio simulation package (VASP), ${ }^{21,22}$ and the details can be found elsewhere. ${ }^{12}$

Figure 1 shows the $\theta-2 \theta$ x-ray diffraction scan of an as-grown $\left[\left(\mathrm{BaTiO}_{3}\right)_{6} /\left(\mathrm{SrTiO}_{3}\right)_{5}\right]_{20}$ superlattice. The full widths at half maximum of the 0020 superlattice reflection are $0.26^{\circ}$ and $0.22^{\circ}$ in $2 \theta$ and $\omega$, respectively. These values are close to the instrumental resolution of our Picker fourcircle x-ray diffractometer. The presence and sharpness of the superlattice reflections indicate its long-range structural coherence. The bilayer periodicity of the superlattice determined by a Nelson-Riley analysis ${ }^{23}$ of the $00 \ell$ superlattice reflections is $44.5 \pm 0.2 \AA$, compared to the $43.74 \AA$ of a $\left(\mathrm{BaTiO}_{3}\right)_{6} /\left(\mathrm{SrTiO}_{3}\right)_{5}$ superlattice assuming unstrained $c$-axis $\mathrm{BaTiO}_{3}$ and $\mathrm{SrTiO}_{3}$ lattice constants. A $\phi$ scan of the $101 \underline{1}$ 


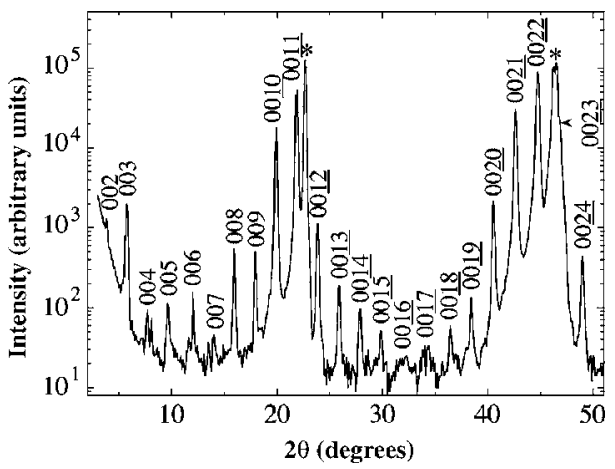

FIG. 1. $\theta-2 \theta$ x-ray diffraction pattern of a $\left[\left(\mathrm{BaTiO}_{3}\right)_{6} /\left(\mathrm{SrTiO}_{3}\right)_{5}\right]_{20}$ superlattice grown on (001) $\mathrm{SrTiO}_{3}$ indicating a superlattice period of $44.5 \pm 0.2$ Å. Substrate peaks are labeled with “*”.

superlattice reflection of the thin film (plot not shown) reveals that the film is epitaxially grown on the (001) surface of $\mathrm{SrTiO}_{3}$ and has an in-plane film-substrate orientation relationship of $\mathrm{BaTiO}_{3}\langle 010\rangle \| \mathrm{SrTiO}_{3}\langle 010\rangle$. From both scans the in-plane lattice constant is determined to be $a=3.91 \pm 0.01 \AA$, resulting in a $c / a$ ratio of $11.38 \pm 0.06$ for the $\left(\mathrm{BaTiO}_{3}\right)_{6} /\left(\mathrm{SrTiO}_{3}\right)_{5}$ supercell.

A careful examination throughout the thin area of a plane-view TEM specimen (images not shown) reveals a linear density of misfit dislocations of $2.9 \times 10^{3} / \mathrm{cm}$, which corresponds to the preservation of approximately $99.5 \%$ of the in-plane lattice mismatch strain in the $\mathrm{BaTiO}_{3}$ layers.

Figure 2 is a cross-sectional HRTEM image of the same $\left[\left(\mathrm{BaTiO}_{3}\right)_{6} /\left(\mathrm{SrTiO}_{3}\right)_{5}\right]_{20}$ superlattice after anneal. The HRTEM images obtained at specific imaging conditions appropriate to the "chemical lattice imaging" technique ${ }^{24}$ re-

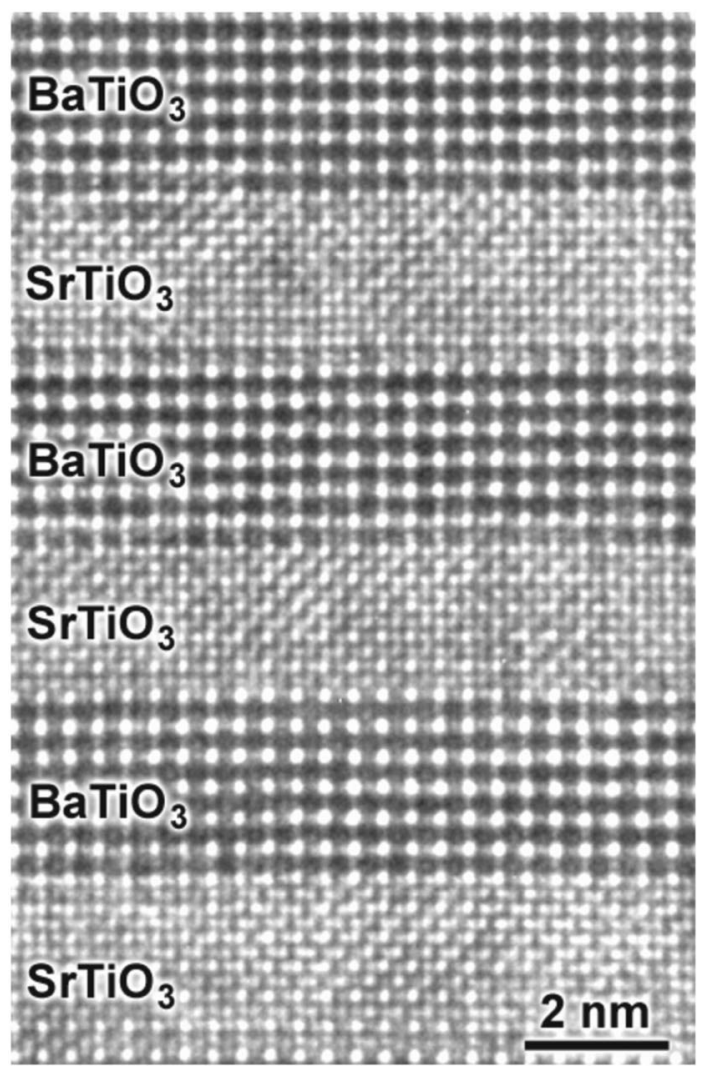

FIG. 2. An HRTEM image of a portion of the same $\left[\left(\mathrm{BaTiO}_{3}\right)_{6} /\left(\mathrm{SrTiO}_{3}\right)_{5}\right]_{20}$ superlattice as analyzed in Fig. 1 with the incident electron beam along the [100] zone axis of $\mathrm{SrTiO}_{3}$.

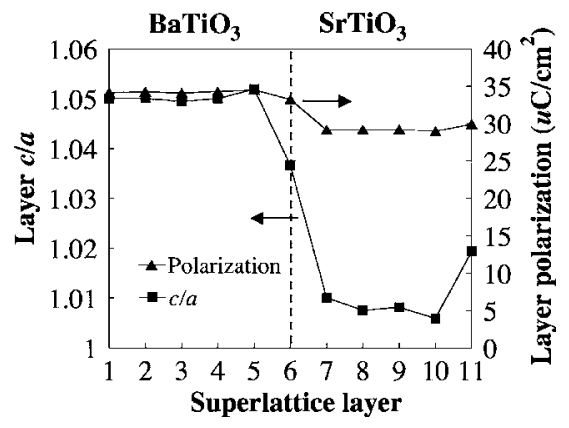

FIG. 3. Local crystallographic $c / a$ ratio and polarization within $\mathrm{BaTiO}_{3}$ and $\mathrm{SrTiO}_{3}$ perovskite unit cells in one period of the superlattice structure based on atomic positions obtained from the first-principles calculation. The vertical dashed line indicates the interface between $\mathrm{BaTiO}_{3}$ and $\mathrm{SrTiO}_{3}$.

veal that no significant interdiffusion of $\mathrm{Ba}^{2+}$ and $\mathrm{Sr}^{2+}$ takes place during annealing. ${ }^{25}$

The crystallographic $c / a$ ratio of the $\mathrm{BaTiO}_{3}$ and $\mathrm{SrTiO}_{3}$ layers was measured by a quantitative analysis of the HRTEM images using Gatan's DIGITALMICROGRAPH software. In the HRTEM images, the positions of the intensity maxima at each barium and strontium atom column (bright spots in Fig. 2) were taken to measure the lattice constant. The measured $c / a$ ratios are $\sim 1.05 \pm 0.02$ and $1.00 \pm 0.01$ for $\mathrm{BaTiO}_{3}$ and $\mathrm{SrTiO}_{3}$, respectively. Note that the $c / a$ ratio of bulk $\mathrm{BaTiO}_{3}$ is 1.013 . A substantial increase of the tetragonality of $\mathrm{BaTiO}_{3}$ is evident. In contrast, the $c / a$ ratio of the $\mathrm{SrTiO}_{3}$ layers remains very close to unity.

Recent experimental results on thin $\mathrm{PbTiO}_{3}$ thin films ${ }^{26}$ and $\mathrm{PbTiO}_{3} / \mathrm{SrTiO}_{3}$ superlattices ${ }^{27}$ suggests that a change in tetragonality is related to a change in polarization. To understand the effect of increased tetragonality upon the polarization of our superlattice, first-principles calculations were performed. The atomic positions were calculated by constraining the in-plane lattice parameter of a periodically repeated $\left(\mathrm{BaTiO}_{3}\right)_{6} /\left(\mathrm{SrTiO}_{3}\right)_{5}$ supercell to the lattice parameter of $\mathrm{SrTiO}_{3}$ determined by first-principles calculations within the local density approximation $(a=3.863 \AA)$ and relaxing the internal structural parameters with the space group $P 4 \mathrm{~mm}$, allowing a nonzero polarization vector parallel to $c$. This procedure predicts $c / a$ of 11.34 for the supercell, in good agreement with the measured value from XRD $11.38 \pm 0.06$.

For each perovskite unit cell layer in one period of the superlattice, we plot in Fig. 3 the calculated local $c / a$ ratio and polarization, the latter estimated using first-principles bulk effective charges. The effects of both strain and electrostatics are clearly evident. As expected from electrostatic arguments, ${ }^{12}$ the polarization appears to be nearly constant throughout the superlattice layers except near the interface, where a somewhat exaggerated discontinuity arises from approximating the interface polarization with bulk effective charges. For the $\mathrm{BaTiO}_{3}$ layers, the average local $c / a$ ratio is computed to be $\sim 1.0503$, and the polarization is $34.27 \mu \mathrm{C} / \mathrm{cm}^{2}$. Both are significantly larger than for unstrained tetragonal $\mathrm{BaTiO}_{3}$, for which we find a calculated $c / a$ ratio of 1.013 , in precise agreement with experiment, and a calculated spontaneous polarization ${ }^{12}$ of $24.97 \mu \mathrm{C} / \mathrm{cm}^{2}$, very close to the measured value ${ }^{28}$ of $26 \mu \mathrm{C} / \mathrm{cm}^{2}$. The calculations also indicate that the $\mathrm{SrTiO}_{3}$ layers are tetragonal with an average $c / a$ ratio of 1.008 . While significant, the deviation from $c / a=1$ of $\mathrm{SrTiO}_{3}$ is too small to be resolved by HRTEM. Summing the contribution 


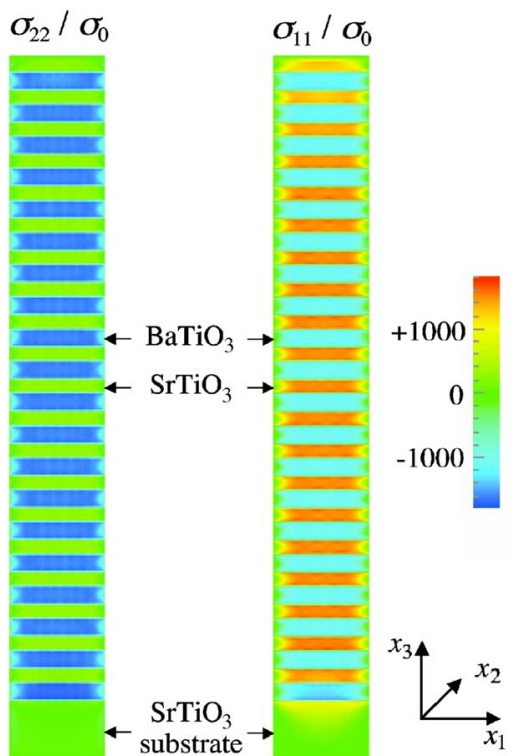

FIG. 4. (Color online) Simulated stresses $\sigma_{22}$ and $\sigma_{11}$ in the TEM specimen of the $\left[\left(\mathrm{BaTiO}_{3}\right)_{6} /\left(\mathrm{SrTiO}_{3}\right)_{5}\right]_{20}$ superlattice calculated using phase-field modeling. The normalization factor is $\sigma_{0}=2.6 \mathrm{MPa}$. Compressive and tensile stresses are indicated by "-" and "+", respectively.

from each layer, we estimate the overall polarization to be $31.2 \mu \mathrm{C} / \mathrm{cm}^{2}$ from the calculation, larger than that of bulk $\mathrm{BaTiO}_{3}$. (A rigorous Berry phase calculation of the superlattice polarization gives $29.375 \mu \mathrm{C} / \mathrm{cm}^{2}$, in excellent agreement with our estimate based on bulk effective charges.)

We note that the stress in the TEM specimen of the superlattice differs from that in the original superlattice due to the thin foil nature of the TEM specimen. To estimate this effect on $c / a$, the stress distribution and the lattice constants of the superlattice in the TEM sample were calculated by phase-field modeling. In the modeling, $\mathrm{BaTiO}_{3}$ and $\mathrm{SrTiO}_{3}$ were assumed to possess their bulk elastic and thermodynamic properties. According to HRTEM image simulations, the thickness of the region imaged in Fig. 2 is in the range of $10-15 \mathrm{~nm}$ in the electron beam direction (denoted by $x_{1}$ ). The axis perpendicular to the surface of the substrate is denoted as $x_{3}$ and the in-plane axis orthogonal to $x_{1}$ is denoted as $x_{2}$. A thin foil with thickness (in the $x_{1}$ direction) of $13.6 \mathrm{~nm}$ and superlattice film height and substrate height of 88 and $100 \mathrm{~nm}$, respectively, in the $x_{3}$ direction was modeled. Periodic boundary conditions were used in the $x_{2}$ direction, implying an infinite length along this direction.

The calculated stress distribution is shown in Fig. 4. One can see that the $\mathrm{BaTiO}_{3}$ layers endure an unrelaxed compressive stress $\sigma_{22}$ due to the underlying $\mathrm{SrTiO}_{3}$ substrate and the $\mathrm{SrTiO}_{3}$ alternative layers and absence of free surface in the $x_{2}$ direction, while $\sigma_{22}$ is close to zero in the $\mathrm{SrTiO}_{3}$ layers. The stress component $\sigma_{11}$, in contrast, is subject to partial relief. Although compressive in the $\mathrm{BaTiO}_{3}$ layers and tensile in the $\mathrm{SrTiO}_{3}$ layers, $\sigma_{11}$ is of the same magnitude in the $\mathrm{BaTiO}_{3}$ layers and the proximate $\mathrm{SrTiO}_{3}$ layers. A nearly $30 \%$ stress relief of $\sigma_{11}$ in the $\mathrm{BaTiO}_{3}$ layers is calculated at the center part of the superlattice thin film. According to the model, the average over the entire TEM foil of the lattice constants of the $\mathrm{BaTiO}_{3}$ and $\mathrm{SrTiO}_{3}$ layers in the superlattice are $a_{1}=3.976 \AA, a_{2}=3.905 \AA$, and $c=4.038 \AA$ for the $\mathrm{BaTiO}_{3}$ layers and $a_{1}=3.958 \AA, \quad a_{2}=3.905 \AA$, and $c=3.879 \AA$ for the $\mathrm{SrTiO}_{3}$ layers, respectively. Note that $a_{1}$ is no longer equal to $a_{2}$ in the TEM specimen. As the HRTEM image is a projection of the crystal lattices along the $x_{1}$ axis, the lattice parameters measured from HRTEM images are $c$ and $a_{2}$. The $c / a_{2}$ ratio of the $\mathrm{BaTiO}_{3}$ layers obtained from modeling is 1.034 , which is slightly smaller than the value measured from the HRTEM images $(1.05 \pm 0.02)$.

The lattice constants of the superlattice for the as-grown film (before it was thinned for TEM) were also calculated by phase field modeling. The resulting $c=44.30 \AA, a=3.905 \AA$, and $c / a=11.34$ are in excellent agreement with the firstprinciples calculations $(c / a=11.34)$ and XRD measurement $(c=44.5 \pm 0.2 \AA, a=3.91 \pm 0.01 \AA$, and $c / a=11.38 \pm 0.06)$.

These results suggest an enhanced polarization in commensurate short-period $\mathrm{BaTiO}_{3} / \mathrm{SrTiO}_{3}$ superlattices.

The authors acknowledge the support of the National Science Foundation through Grant No. DMR-0507146.

${ }^{1}$ S. Li, J. A. Eastman, J. M. Vetrone, R. E. Newnham, and L. E. Cross, Philos. Mag. B 76, 47 (1997).

${ }^{2}$ N. Sai, B. Meyer, and D. Vanderbilt, Phys. Rev. Lett. 84, 5636 (2000).

${ }^{3}$ T. Shimuta, O. Nakagawara, T. Makino, S. Arai, H. Tabata, and T. Kawai, J. Appl. Phys. 91, 2290 (2002).

${ }^{4}$ M. H. Corbett, R. M. Bowman, J. M. Gregg, and D. T. Foord, Appl. Phys. Lett. 79, 815 (2001).

${ }^{5}$ L. Kim, D. Jung, J. Kim, Y. S. Kim, and J. Lee, Appl. Phys. Lett. 82, 2118 (2003).

${ }^{6}$ H.-M. Christen, L. A. Knauss, and K. S. Harshavardhan, Mater. Sci. Eng., B 56, 200 (1998).

${ }^{7}$ H. M. Christen, E. D. Specht, S. S. Silliman, and K. S. Harshavardhan, Phys. Rev. B 68, 020101 (2003).

${ }^{8}$ M. R. Warusawithana, E. V. Colla, J. N. Eckstein, and M. B. Weissman, Phys. Rev. Lett. 90, 036802 (2003).

${ }^{9}$ H. N. Lee, H. M. Christen, M. F. Chisholm, C. M. Rouleau, and D. H. Lowndes, Nature (London) 433, 395 (2005).

${ }^{10}$ D. O'Neill, R. M. Bowman, and J. M. Gregg, Appl. Phys. Lett. 77, 1520 (2000).

${ }^{11}$ G. Catalan, D. O'Neill, R. M. Bowman, and J. M. Gregg, Appl. Phys. Lett. 77, 3078 (2000).

${ }^{12}$ J. B. Neaton and K. M. Rabe, Appl. Phys. Lett. 82, 1586 (2003).

${ }^{13}$ A. F. Devonshire, Philos. Mag., Suppl. 3, 85 (1954).

${ }^{14}$ H. Uwe and T. Sakudo, Phys. Rev. B 13, 271 (1976).

${ }^{15}$ J. H. Haeni, P. Irvin, W. Chang, R. Uecker, P. Reiche, Y. L. Li, S. Choudhury, W. Tian, M. E. Hawley, B. Craigo, A. K. Tagantsev, X. Q. Pan, S. K. Streiffer, L. Q. Chen, S. W. Kirchoefer, J. Levy, and D. G. Schlom, Nature (London) 430, 758 (2004).

${ }^{16}$ T. M. Shaw, A. Gupta, M. Y. Chern, P. E. Baston, R. B. Laibowitz, and B. A. Scott, J. Mater. Res. 9, 2566 (1994).

${ }^{17}$ Z. Y. Wang, T. Yasuda, S. Hatatani, and S. Oda, Jpn. J. Appl. Phys., Part 1 38, 6817 (1999)

${ }^{18}$ J. C. Jiang, X. Q. Pan, W. Tian, C. D. Theis, and D. G. Schlom, Appl. Phys. Lett. 74, 2851 (1999).

${ }^{19}$ D. G. Schlom, J. H. Haeni, J. Lettieri, C. D. Theis, W. Tian, J. C. Jiang, and X. Q. Pan, Mater. Sci. Eng., B 87, 282 (2001).

${ }^{20}$ J. H. Haeni, C. D. Theis, and D. G. Schlom, J. Electroceram. 4, 385 (2000).

${ }^{21}$ G. Kresse and J. Furthmuller, Phys. Rev. B 54, 11169 (1996).

${ }^{22}$ G. Kresse and D. Joubert, Phys. Rev. B 59, 1758 (1999).

${ }^{23}$ J. B. Nelson and D. P. Riley, Proc. Phys. Soc. London 57, 160 (1945).

${ }^{24}$ A. Ourmazd, F. H. Baumann, M. Bode, and Y. Kim, Ultramicroscopy 34, 237 (1990).

${ }^{25}$ W. Tian, Ph.D. thesis, University of Michigan, 2002.

${ }^{26}$ C. Lichtensteiger, J.-M. Triscone, J. Junqura, and P. Ghosez, Phys. Rev. Lett. 94, 047603 (2005).

${ }^{27}$ M. Dawber, C. Lichtensteiger, M. Cantoni, M. Veithen, P. Ghosez, K. Johnston, K. M. Rabe, and J.-M. Triscone, Phys. Rev. Lett. 95, 177601 (2005).

${ }^{28}$ G. Shirane, H. Danner, and P. Pepinsky, Phys. Rev. 105, 856 (1957). 